\title{
Conceptual relation preference: A matter of strategy or one of salience?
}

Olivera, Savic; Thierry, Guillaume; Kovic, Vanja

\section{Acta Psychologica}

DOI:

10.1016/j.actpsy.2020.103018

Published: 01/03/2020

Peer reviewed version

Cyswllt i'r cyhoeddiad / Link to publication

Dyfyniad o'r fersiwn a gyhoeddwyd / Citation for published version (APA):

Olivera, S., Thierry, G., \& Kovic, V. (2020). Conceptual relation preference: A matter of strategy or one of salience? Acta Psychologica, 204, [103018].

https://doi.org/10.1016/j.actpsy.2020.103018

\section{Hawliau Cyffredinol / General rights}

Copyright and moral rights for the publications made accessible in the public portal are retained by the authors and/or other copyright owners and it is a condition of accessing publications that users recognise and abide by the legal requirements associated with these rights.

- Users may download and print one copy of any publication from the public portal for the purpose of private study or research.

- You may not further distribute the material or use it for any profit-making activity or commercial gain

- You may freely distribute the URL identifying the publication in the public portal ?

Take down policy

If you believe that this document breaches copyright please contact us providing details, and we will remove access to the work immediately and investigate your claim. 


\title{
Conceptual relation preference: A matter of strategy or one of salience?
}

\author{
Olivera Savic, Guillaume Thierry and Vanja Kovic
}

\begin{abstract}
In order to determine whether preference in object matching tasks measures participants' strategy or tells us something about the salience of relations between corresponding concepts, we conducted three experiments. In Experiment 1 and Experiment 2, we approached this question by measuring the ease with which adult participants process different relations when they are under strategic instruction. When asked to group objects based on thematic or taxonomic relatedness, participants were slower (Experiment 2) and tended to make more errors (Experiment 1-2) when they had to find a taxonomically related pair than when they searched for a thematically related one. In Experiment 3, participants performed a standard matching task and their eye-movements were monitored throughout. In addition to the strong thematic preference in participants' choices, we measured longer fixations to thematically related objects than taxonomic competitors. Even though thematic and taxonomic information appear to compete for selection in early phases of observation, thematic conceptual relations appear to be more salient and preferred, independently of instruction.
\end{abstract}

Key words: Matching-to-sample task; Thematic relations; Taxonomic relations; Eyetracking; Thematic preference 


\section{Conceptual relation preference: A matter of strategy or one of salience?}

Olivera Savic ${ }^{\mathrm{a}, \mathrm{b}}$, Guillaume Thierryc and Vanja Kovic ${ }^{\mathrm{a}}$

Affiliations

${ }^{a}$ Laboratory for Neurocognition and Applied Cognition, Department of Psychology,

Faculty of Philosophy, University of Belgrade, 18-20 Čika Ljubina, Belgrade, Serbia

${ }^{b}$ Department of Psychology, The Ohio State University, 1835 Neil Ave, 43210, Columbus, Ohio, USA

${ }^{\mathrm{c}}$ School of Psychology, Bangor University, Adeilad Brigantia, Bangor, Gwynedd LL57

2AS, UK

* Correspondence:

Olivera Savić, savic.7@osu.edu 


\begin{abstract}
In order to determine whether preference in object matching tasks measures participants' strategy or tells us something about the salience of relations between corresponding concepts, we conducted three experiments. In Experiment 1 and Experiment 2, we approached this question by measuring the ease with which adult participants process different relations when they are under strategic instruction. When asked to group objects based on thematic or taxonomic relatedness, participants were slower (Experiment 2) and tended to make more errors (Experiment 1-2) when they had to find a taxonomically related pair than when they searched for a thematically related one. In Experiment 3, participants performed a standard matching task and their eye-movements were monitored throughout. In addition to the strong thematic preference in participants' choices, we measured longer fixations to thematically related objects than taxonomic competitors. Even though thematic and taxonomic information appear to compete for selection in early phases of observation, thematic conceptual relations appear to be more salient and preferred, independently of instruction.
\end{abstract}

Key words: Matching-to-sample task; Thematic relations; Taxonomic relations; Eyetracking; Thematic preference 


\section{Conceptual relation preference: A matter of strategy or one of salience?}

\section{Introduction}

In a standard matching-to-sample task, participants are shown a target object and two or more optional objects and they are asked to report which of the optional objects are similar or go together with the target (e.g., Smiley \& Brown, 1979; Lin and Murphy 2001). In order to perform the task, participants need to identify a criterion on the basis of which to define the grouping. For example, given a picture of a cow as target and pictures of a donkey and milk as options, a participant may choose to group cow and donkey together because they are both animals or she may group cow and milk because cows produce milk.

There is a long-held belief that thematic categorization style, that is, the grouping of objects based on complementary, functional relations and contextual co-occurrences (e.g. cow - milk, horse - saddle), reflects a more basic and unsophisticated form of reasoning rooted in episodic knowledge and that it dominates in early childhood (see for instance, Vygotsky, 1962; Inhelder \& Piaget, 1964; Lucariello, Kyratzis, \& Nelson, 1992). The idea is that through learning and development categorization gradually becomes taxonomic (e.g. cow-donkey, horse - zebra), that is, based on shared properties and attributes, information that is formal and mostly context-free. Such hypothetical course of cognitive development involving a shift from thematic to taxonomic reasoning has its roots in the most influential theories of cognitive development (Vygotsky, 1962; Inhelder \& Piaget, 1964) and has received supporting evidence across a wide range of cognitive tasks. It has been conceptualized as a shift from syntagmatic to paradigmatic (Brown \& Berko, 1960; Ervin, 1961; Nelson, 1977) or functional to conceptual style (Moran, Mefford \& Kimble, 1964) in word associations, preference for complementary vs. similarity relationships in memory tasks (Denney \& Ziobrowsky, 1972), and, finally, as a thematic-to-taxonomic shift in matching tasks (Smiley \& Brown, 1979).

The strong version of the thematic-to-taxonomic shift hypothesis that assumes a fundamental change in the way semantic knowledge is organized (e.g. Inhelder \& Piaget, 
1964) has been questioned early on. A number of studies has argued that children are capable of both thematic and taxonomic categorization long before the age of 7 (Smiley \& Brown, 1979; Markman, Cox, \& Machida, 1981; Waxman \& Gelman, 1986; Kalénine \& Bonthoux, 2006) and that children's preference to group objects thematically may not necessarily imply lack of access to taxonomic knowledge (Smiley \& Brown, 1979; Denney \& Ziobrowsky, 1972; Waxman \& Gelman, 1986). Interestingly, much of the discussion around a thematic-to-taxonomic shift has focused on the issue of thematic conceptual preference in young children, while the idea that older children and adults tend to prefer taxonomic relationship has seldom been questioned until recently.

In a series of experiments, Lin and Murphy (2001) have shown that preferences in matching tasks depend on instructions and stimulus characteristics, but that adults, contrary to the prediction of the thematic-to-taxonomic shift hypothesis, tend to rely also on thematic relations in matching tasks (49\%-70\% of thematic responses across 10 experiments). These results could not be explained by a lack of education or peculiarities of culture or language, as was earlier proposed to account for scattered findings of adult thematic sorting (Luria, 1976; Chiu, 1972; Ji, Zhang, \& Nisbett, 2004), since their participants were young educated native speakers of English.

Findings of adults' thematic preference (Lin \& Murphy, 2001; Murphy, 2001; for review see Estes, Golonka, \& Jones, 2011) have rekindled interest in studying the hierarchy of thematic and taxonomic relations and questioned the existence of a conceptual shift, suggesting that, similar to the view of Shank (1976), it could be that at all ages thematic relations are more salient than hierarchical, taxonomic ones. Recent studies that investigated temporal dynamics of thematic and taxonomic knowledge support this hypothesis. It has been demonstrated that even in adults thematic relations may be detected earlier than relations based on category membership (Kalénine, Mirman, Middleton, \& Buxbaum, 2012; Pluciennicka, Coello, \& Kalénine, 2013) and that semantic integration of thematic information may be easier and less effortful than integration of taxonomic information (Chen et al, 2013; Savic, Savic, \& Kovic, 2017). While these recent studies provide evidence on thematic and taxonomic relations having different time courses of 
activation and different neural correlates (for a recent review see Mirman, Landrigan, \& Britt, 2017), there seems to be little doubt that both thematically and taxonomically related concepts are automatically activated during early semantic processing.

These conflicting results have brought into question the usefulness of the matching task for measuring conceptual preference. In other words, to what extent do categorization preferences index cognitive strategies deriving from cultural (e.g. schooling context) or immediate (e.g. instruction comprehension) context ${ }^{1} \mathrm{~s}$ and to what extent do they tell us something about the structure of long-term memory and conceptual organization?

Here, we attempted to tease apart the potential strategic and conceptual effects in relation preference of adults in object matching tasks. We addressed this problem in two ways. In the first two experiments, we approached this question by measuring the ease with which adult participants process different relations when they are under strategic instruction. We employed a modified version of matching-to-sample task in which participants were guided to group objects based on thematic relatedness, taxonomic relatedness, or neither. If conceptual preference derives solely from cognitive strategies employed by the participant, she should be equally accurate and fast when required to match objects on a thematic or a taxonomic basis. In contrast, if the participant's performance is better in one condition than the other, the results would favor a difference in conceptual organization.

In the third experiment, we combine the traditional matching-to-sample task with eye-tracking. Tracking of participants' attention allows us to shed light on dynamic aspects of categorization during object pairing and address the question of difference in the salience

\footnotetext{
${ }^{1}$ For example, understanding of the instructions may make participants to intentionally respond in thematic manner although they were perhaps initially inclined towards the taxonomic option. This would be considered immediate contextual effect.

In addition to the immediate context bias, other authors have argued that preferences in matching tasks may be explained by specific cultural practices (e.g. Nisbett \& Miyamoto, 2005), peculiarities of different languages and writing system (e.g. Ji, Zhang \& Nisbett, 2004), or expectations formed by formal education.
} 
of different semantic relatedness types (Huettig et al, 2006; Yee, Overton \& ThopmsonSchill, 2009).

All experiments were conducted on the same set of stimuli, with all participants being educated adult native speakers of Serbian.

\section{Experiment 1: Guided matching task}

Experiment 1 employed a modified matching-to-sample task. In addition to the main elements of the standard matching-to-sample task: base object and (two or more) option objects, we introduced instructions guiding participants choice, i.e., we engaged them in a guided matching task. There were three versions of the task, differing based on when instructions were introduced:

Experiment 1a: Randomized matching- instruction precedes prime

Experiment 1b: Blocked matching - instruction precedes block of trials

Experiment 1c: Randomized matching - prime precedes instructions

The reasoning behind Experiment 1a was that having the instruction between the base and the option object may strongly reduce the priming effects, so instructions were introduced first, followed by prime (base) and target objects. In Experiment 1b, we used a blocked design in order to reduce potential switching effects. Since Experiment 1a has an additional memory component (participants need to remember the instructions while listening to the auditory prime/base) and that it is likely easier for participants to keep in mind a familiar word (auditory prime) than the task instructions, we changed the trial structure, by presenting auditory prime first and then the task instructions in Experiment 1c.

\section{Methods}

\section{Participants}


The participants were second-year undergraduate psychology students, all native speakers of Serbian. They gave written informed consent prior to the study. We tested 29 individuals in Experiment 1a, 35 in Experiment 1b and 24 in Experiment 1c.

\section{Stimuli}

Picture stimuli were 78 high-quality photographs of real objects chosen from the Hemera image database (Hemera, 2000), The Hatfield Image Test (Adlington, Laws, \& Gale, 2008), and commercial websites. All the images were of the same size and all of them had a white background. Images had a maximum size of $5 \times 5 \mathrm{~cm}$ and were scaled so that at least one of the two dimensions was $5 \mathrm{~cm}$ on a 15.6-inch screen. All stimuli depicted highly familiar objects easily recognizable when presented in isolation on a white background.

Auditory stimuli were 26 object labels pronounced at a normal speaking rate by a native adult male speaker of Serbian and recorded in the context of a carrier phrase: 'Say $<$ label $>$, please.' so as to minimize risks of abnormal durations and unnatural prosody when words are produced in isolation. This procedure is widely used in studies of auditory lexical decision (see Slowiaczek \& Pisoni, 1986). Sound signals corresponding to each label were spliced out from the carrier phrase, edited to remove background noise using Praat software (Boersma \& Weenink, 2009), and presented as single auditory word stimuli. Auditory stimulus duration varied from $365 \mathrm{~ms}$ to $956 \mathrm{~ms}$. In order to standardize stimulus duration, silence was added at the end of all sound files, so that they all lasted $1500 \mathrm{~ms}$.

Stimuli were selected based on previous studies examining differences in thematic and taxonomic processing (Chiu, 1972; Smiley \& Brown, 1979; Osborne \& Calhoun, 1998; Lin \& Murphy, 2001; Unsworth, Sears, \& Pexman, 2005; Kalénine \& Bonthoux, 2006). The strength of thematic and taxonomic relatedness of the stimuli pairs selected for this study was based on relatedness judgment studies conducted on a sample of Serbian adults (Ilic, 2015). Two separate groups of participants were asked to judge the extent to which the items in each pair were either thematically related or taxonomically related, using a 7point scale. The instruction were based on the definitions of thematic and taxonomic categories found in Estes et al. (2011). Thematic pairs in our sample were more strongly 
thematically related than taxonomic pairs $(t(23)=8.34, p<.001)$ and taxonomic pairs were more strongly taxonomically related than thematic pairs $(t(23)=16.59, p<.001)$, confirming the taxonomic-thematic distinction between the stimuli pairs. The instructions and the complete list of stimuli can be found in Table 1 in Appendix. From the 26 stimuli sets created, 2 were used as practice and 24 as test sets.

\section{Design}

The experiments were run with Task (Task: Thematic; Taxonomic; Unrelated) as a within-subject factor. In Experiment 1a each participant received a random order of 72 experimental trials, each of the 24 triads being presented 3 times, once per task. In Experiment 1b, participants completed 3 thematic, 3 taxonomic and 3 unrelated item search blocks (72 trials). Each block begun with task instructions (e.g. "find the THEMATICALLY related option") followed by presentation of 8 trials (Illustration 1). The set of 24 triads was

divided into three groups. Each group was presented three times, each time in the context of one of the three tasks. The presentation order of the blocks was quazi-randomized, so that the two blocks with the same task, or two blocks containing same stimuli would never succeed each other. In Experiment, 1c participants received a random order of 24 experimental trials, one third of the trials with thematic, one with taxonomic, and one with unrelated instructions. The assignment of the stimuli to the task was counterbalanced across participants.

\section{Procedure}

Before the start of the experiment, participants received definitions of thematic and taxonomic relationships with accompanying examples. The instructions were based on the definitions of thematic and taxonomic categories found in Estes et al. (2011). Thematic relationship was described as temporal, spatial, functional, or causal relationship between the objects that have complementary roles in the same scenario or event. Taxonomically related objects were defined as semantically related objects that share features and hence belong to the same category. Both definitions were followed by 2 examples (thematic: plane - pilot, flower - vase; taxonomic: plane - train, flower - tree). 
Each trial of Experiment 1a began with the instruction, written on the screen (e.g. "find the THEMATICALLY related option") for $2500 \mathrm{~ms}$. The instruction was followed by presentation of a fixation cross for $500 \mathrm{~ms}$, which remained on the screen during presentation of the auditory prime word $(1500 \mathrm{~ms})$. After auditory word prime presentation, pictures of three objects (one thematically, one taxonomically related, and one unrelated) were presented on the same screen in the angles of an imagined triangle (Figure 1). Object location assignment (top, left, right) was rotated across trials. Picture stimuli were presented on the standard 15-inch monitor of a laptop computer (refresh rate of $60 \mathrm{~Hz}$ ) using Superlab 4.0 software (Cedrus Corporation). The approximate viewing distance was $60 \mathrm{~cm}$. Stimuli remained on the screen until participant responded. No feedback was given. Participants responded by pressing designated keys on a keyboard. Accuracy and reaction times (recorded from the onset of the visual stimuli) were recorded for each trial.

In contrast to Experiment 1a, Experiment $1 \mathrm{~b}$ instructions were presented only once before each set of 8 trials. The procedure of Experiment $1 \mathrm{c}$ matched the one described for Experiment 1a with one difference only - the task instruction and the auditory prime were presented in the reverse order so that the auditory prime preceded instruction (Figure 1). 


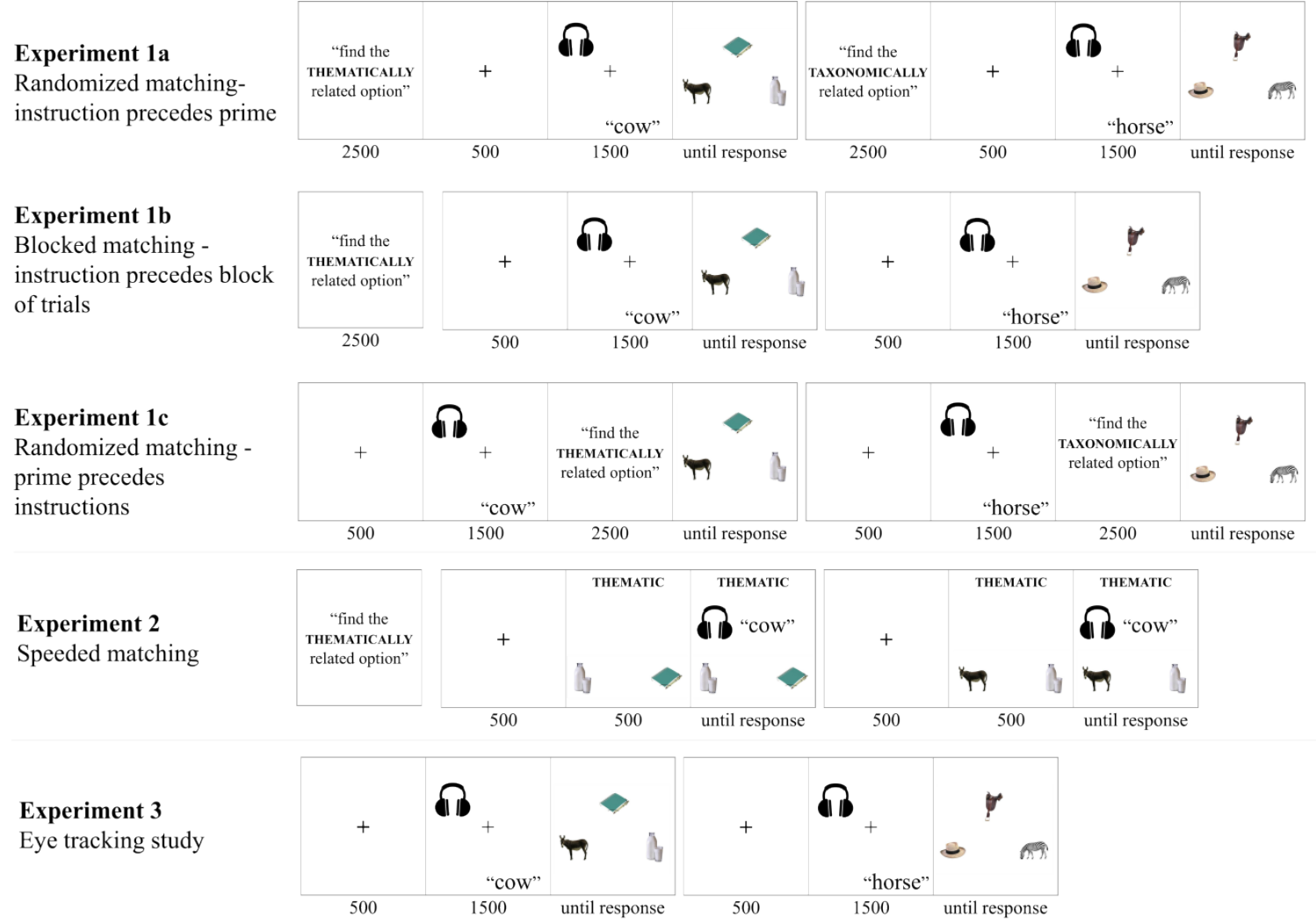

Figure 1: The structure of experimental trials across the three experiments

\section{Results and Discussion}

In order for participant's data to be included in the analysis, average accuracy in the thematic and the taxonomic instruction conditions needed to be above 0.6. Participants who did not reach this criterion were considered not to have learnt the difference between the relationship types or not being able to keep track of the task change during the experiment. Data of two participants from Experiment 1a and 7 participants from Experiment 1b were excluded based on this criterion.

The average accuracy across the three experiments and the three tasks is given in Table 1. Across all three experiments, participants tended to be most accurate when 
looking for Unrelated pairs, but also tended to reach higher level of accuracy in the Thematic than the Taxonomic condition. The effect of the Task reached significance in a repeated measures ANOVA with Task as a within-subject and Experiment as a betweensubject factor $\left(F(2,152)=9.13, p<.001, \eta^{2}=.107\right)$. Pairwise comparisons revealed that participants were less accurate in Taxonomic than in Unrelated and thematic conditions ( $p s$ $<.05$ ), but there was no difference in accuracy between Thematic and Unrelated conditions (Bonferroni's adjustment for multiple comparisons was applied). Accuracy did not differ significantly between experiments, and there was no significant interaction $(p s>.10){ }^{2}$

Table 1. Experiment 1, Accuracy Means and Standard Deviations across the three tasks

\begin{tabular}{|c|c|c|c|c|c|c|c|c|}
\hline & \multicolumn{2}{|c|}{ thematic } & \multicolumn{2}{|c|}{ taxonomic } & \multicolumn{2}{|c|}{ unrelated } & \multicolumn{2}{|c|}{$\begin{array}{l}\text { average by } \\
\text { experiment }\end{array}$} \\
\hline & M & SD & M & SD & M & SD & M & SD \\
\hline Exp1a & .89 & .31 & .88 & .33 & .91 & .29 & .89 & .30 \\
\hline Exp1b & .90 & .31 & .87 & .34 & .92 & .28 & 89 & .31 \\
\hline Exp1c & .94 & .24 & .88 & .33 & .96 & .19 & .93 & .26 \\
\hline $\begin{array}{l}\text { average } \\
\text { by } \\
\text { task* }\end{array}$ & .90 & .30 & .88 & .33 & .92 & .27 & & \\
\hline
\end{tabular}

*number of participants by task was taken into account.

Further analyses of participants' errors showed that most of the errors in the Thematic instruction condition were Taxonomically related items, and most of the errors in the Taxonomic instruction condition were Thematically related items (Table 2).

Table 2. The proportion of errors per type across the three tasks.

\section{task}

type thematic taxonomic Unrelated

\footnotetext{
${ }^{2}$ Errors were spread evenly across stimulus sets in all three tasks, and there were no extreme outliers. The distributions of accuracy rates across stimuli sets in the three tasks is shown in Figure 1 in Appendix.
} 


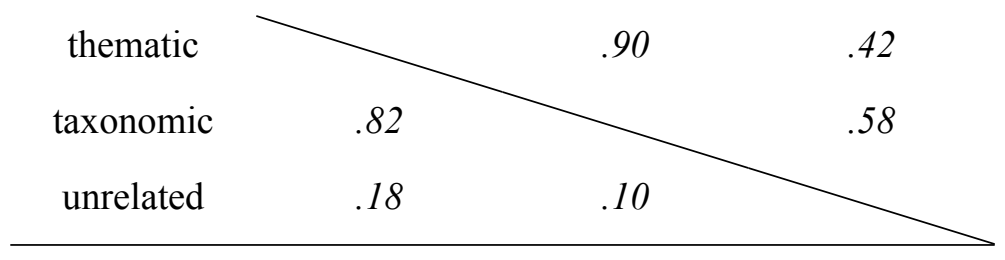

Extremely short and extremely long latencies (exceeding $2.5 \mathrm{SD}$ ) were excluded from the reaction times analysis. A repeated measures ANOVA with Task as within-subject and Experiment as between-subject factor run on log-transformed RTs in correct trials revealed a significant Task main effect $\left(F(4,152)=3.85, p=.023, \eta^{2}=.048\right)$ and significant Task by Experiment interaction $\left(F(4,152)=3.05, p=.019, \eta^{2}=.074\right)$, but no main effect of Experiment. The follow-up analysis showed that the effects were driven by the difference between Thematic and Unrelated condition in Experiment 1c $(p=.019$, after Bonferroni's adjustment for multiple comparisons). Other differences between the conditions were not significant (all $p s>.10)$.

Table 3. Experiment 1, Reaction time Means and Standard Deviations across the three tasks

\begin{tabular}{|c|c|c|c|c|c|c|c|c|}
\hline & \multicolumn{2}{|c|}{ thematic } & \multicolumn{2}{|c|}{ taxonomic } & \multicolumn{2}{|c|}{ unrelated } & \multicolumn{2}{|c|}{$\begin{array}{l}\text { average by } \\
\text { experiment }\end{array}$} \\
\hline & M & SD & M & SD & M & SD & M & SD \\
\hline Exp1a & 1745 & 747 & 1731 & 851 & 1668 & 604 & 1679 & 739 \\
\hline Exp1b & 1588 & 673 & 1508 & 646 & 1571 & 567 & 1556 & 630 \\
\hline Exp1c & 1661 & 995 & 1709 & 998 & 1847 & 812 & 1741 & 937 \\
\hline $\begin{array}{l}\text { average } \\
\text { by task }\end{array}$ & 1665 & 758 & 1630 & 796 & 1649 & 627 & & \\
\hline
\end{tabular}

In summary, statistical analyses presented here speak in favor of thematic matching being easier than taxonomic matching. Although we did not find differences in speed, preference for thematic processing resulted in higher accuracy when participants were asked to match objects based on thematic rather than taxonomic relationships. 


\section{Experiment 2: Speeded guided matching task}

As was explained in the introduction, the salience of the competing conceptual relation in matching-to-sample task should influence the ease with which the target relationship is processed. Thus, if thematic relations are more salient than taxonomic relations, participants should find it more demanding (and thus be slower and less accurate) when matching objects taxonomically. Results of Experiment 1 suggest that indeed reliance on taxonomic relations may be harder than reliance on thematic relations, as participants made more errors when mapping taxonomically related items. However, differences in processing were not detectable in reaction times.

One possible explanation for why we failed to detect differences in speed between thematic and taxonomic task in Experiment 1 may be that the task pace was too slow for subtle differences in speed to be detected. Although participants were encouraged to respond "as fast and as accurately as possible", in order to make sure that they had enough time to respond in a fairly demanding task, there was no built-in time limit. This resulted in long average response times (1630 ms - $1665 \mathrm{~ms})$ and may have led to heightened variance in participants' reaction times diluting subtle differences in the speed of processing, which are difficult (and perhaps impossible) to capture in such conditions.

To overcome these problems we designed Experiment 2. In Experiment 2, we speeded the task, both by shortening presentation time and by providing participants with feedback if it took them more than $800 \mathrm{~ms}$ to respond. In addition, we simplified the experimental design by giving participants two, instead of three, options at a time. To reduce the cost of switching between the tasks, we used blocked design. To further lower working memory demand, we reduced the number of items participants needed to keep in working memory, by changing the order of presentation: Participants first saw two images (e.g. donkey and milk) together with a word reminding them of the task (e.g. thematic) and then heard the prime word (e.g. cow). The images and the task reminder remained on the screen during prime word presentation (see Figure 2). Thus, all of the information needed 
for participant to make a response: task reminder, two options (images) and prime word, were present at the same time.

\section{Participants}

We recruited 38 undergraduate students, all native speakers of Serbian, with normal hearing and normal or corrected-to-normal vision. All participants gave written informed consent prior to the study.

\section{Stimuli and Design}

We used the same stimuli set as the one described in Experiment 1 (Table 1 in Appendix). The design of Experiment 2 was based on the logic of Experiment 1, but differed in several important ways. Participants were instructed to find either thematically or taxonomically related item (Task: Thematic; Taxonomic), in one of the two test conditions (Condition: Neutral; Conflict). In the Neutral condition, participants chose between two options, one that was related (either thematically or taxonomically) and one that was unrelated. In the Conflict condition, both options were related, one thematically and one taxonomically (Figure 2).

The stimuli used in Experiment 1 were rearranged in Experiment 2 so that for each prime word there were 3 pairs of images. For example, set cow: milk-donkey - notebook, where cow is the prime word, was rearranged in sets: A. cow: milk-notebook (Neutral condition; used in Thematic task); B. cow: donkey - notebook (Neutral condition; used in Taxonomic task); and C: cow: milk - donkey (Conflict condition; used in Thematic and Taxonomic task).

Each participant saw all stimuli sets across both Tasks and Conditions, which resulted in total of 96 experiment trials per participant (24 stimuli sets x 2 tasks x 2 conditions). Experiment trials were presented in four blocks: 2 Thematic task blocks and 2 Taxonomic task blocks. The presentation order of the blocks was quazi-randomized, so that the two blocks with the same task would never succeed each other. Presentation order 
within each block was randomized and position of the correct option (left vs. right) was counterbalanced throughout.

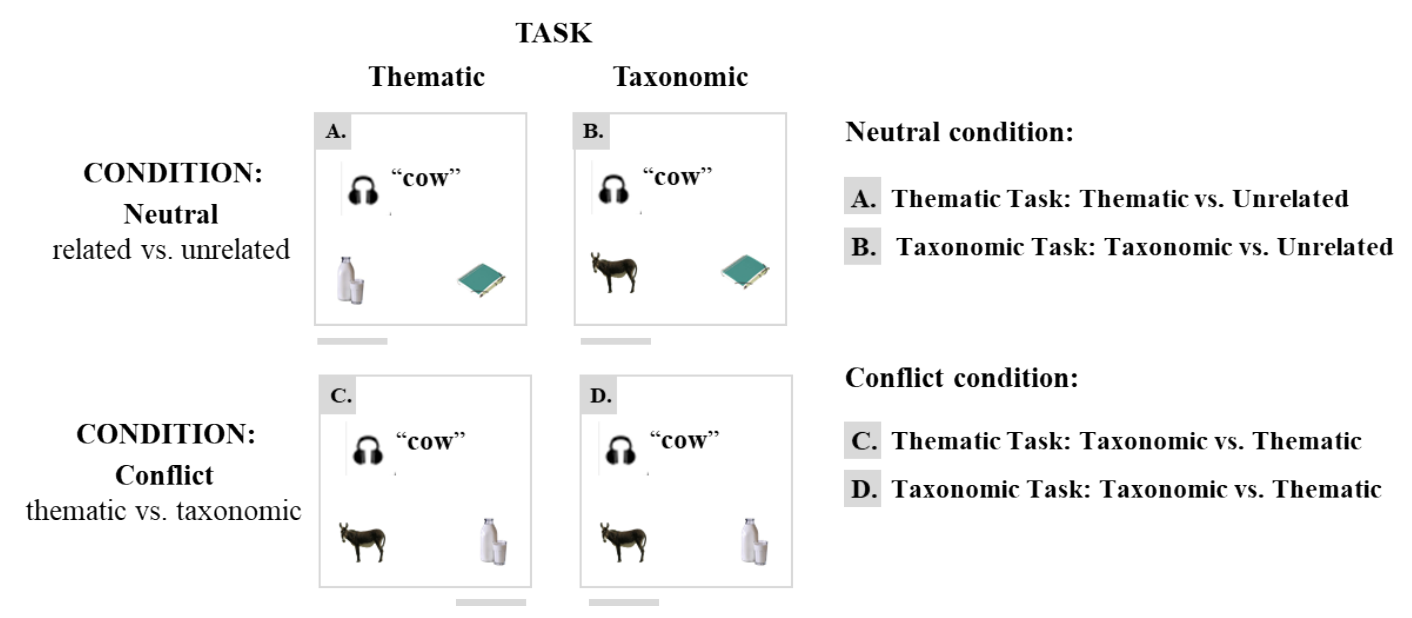

Figure 2: Illustration of the design of Experiment 2.

\section{Procedure}

As in Experiment 1, in Experiment 2 participants were first presented with definitions of thematic and taxonomic relations. We used the same instructions, definitions and examples as used in Experiment 1. After participants learned the concepts of thematic and taxonomic relations, they received 16 training and 96 experiment trials. Experiment trials were presented in 4 blocks: 2 Thematic task and 2 Taxonomic task blocks.

Each block started with an instruction screen ("find the THEMATICALLY related option" or "find the TAXONOMICALLY related option"), followed by a set of 24 experiment trials. Although the task did not change during a given block, a word serving as a task reminder (i.e. "THEMATIC" or "TAXONOMIC") was presented on the top of the screen throughout the experiment (see Figure 1). Each trial began with a fixation cross, presented in the center of the screen for $500 \mathrm{~ms}$. Next, two images (and the task reminder) were presented for $500 \mathrm{~ms}$, after which participants heard auditory prime word (Figure 1). 
Critically, task reminder and option images remained on the screen while participants were presented with the auditory prime. Participants responded by pressing designated keys on a computer keyboard. Accuracy and reaction times (recorded from the onset of the auditory

prime) were recorded for each trial. Participants were not provided with feedback regarding the accuracy of their choice, but they received feedback ("try to be faster") if their response time was longer than $800 \mathrm{~ms}$. All visual stimuli were presented on the standard 15-inch monitor of a laptop computer (refresh rate of $60 \mathrm{~Hz}$ ) using Superlab 4.0 software (Cedrus Corporation), at the approximate viewing distance of $60 \mathrm{~cm}$.

\section{Results and Discussion}

In order for participant's data to be included in the analysis, average accuracy needed to be above 0.6. All participants reached this criterion.

Participant's average accuracy was high $(M=.90, S D=.06)$. However, a repeated measures ANOVA with Task (Thematic vs. Taxonomic) and Condition (Neutral vs. Conflict) as within-subject factors revealed significant main effects of both factors (Task: $F(1,37)=11.85, p<.001, \eta^{2}=.243$; Condition: $\left.F(1,37)=95.03, p<.001, \eta^{2}=.720\right)$ and a significant interaction $\left(F(1,37)=15.60, p<.001, \eta^{2}=.297\right)$. As can be seen on the left panel of Figure 3, participants were equally accurate in finding Thematic and Taxonomic option when the competitor was an Unrelated item. However, when two related options were put in conflict, participants were more accurate in the Thematic than in the Taxonomic task.

Reaction time analysis was further conducted on correct responses. We performed initial data trimming (reaction times larger than $2 \mathrm{~s}$ ) and removing extremely short and extremely long latencies (exceeding $2.5 \mathrm{SD}$ ). A repeated measures ANOVA with Task (Thematic vs. Taxonomic) and Condition (Neutral vs. Conflict) as within-subject factors, run on log-transformed reaction times, revealed a significant main effect of Task $(F(1,37)$ $\left.=5.57, p=.024, \eta^{2}=.131\right)$. Main effect of Condition and the interaction of two factors was not significant $(p s>.10, F s<1)$. Participants were faster in Thematic than in the Taxonomic task across both Neutral $(d=35.5 \mathrm{~ms})$ and Conflict condition $(d=37.8 \mathrm{~ms})$. 
a.

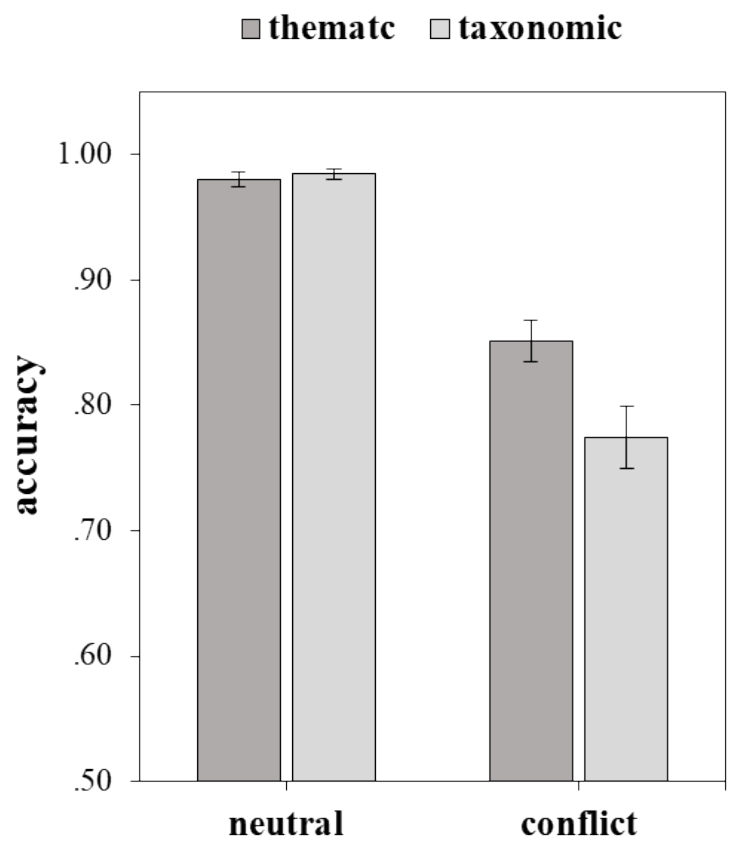

b.

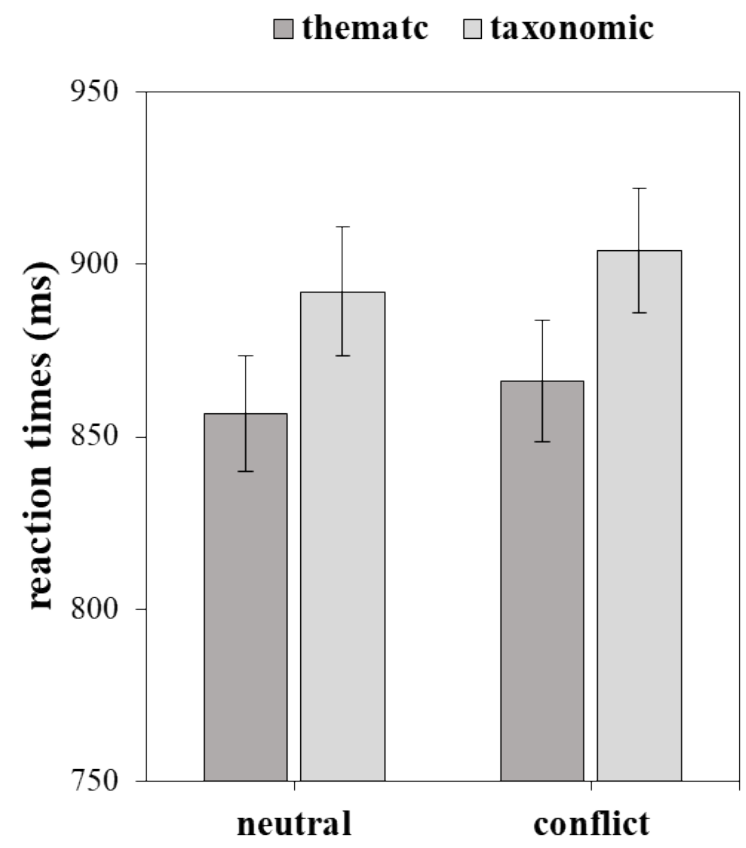

Figure 3. Average accuracy (left) and average reaction times (right) in thematic and taxonomic task across the two conditions: neutral and conflict. Error bars represent standard errors of mean.

To summarize, Experiment 2 provides further support for thematic conceptual preference in adulthood. Although participants were equally accurate when searching for Thematically and Taxonomically related items in Neutral condition, i.e. when the competitor was an Unrelated item, they made more errors in Taxonomic than in Thematic task when Thematic and Taxonomic items directly competed against each other (Conflict condition). In other words, although both Thematic and Taxonomic competitor in Conflict condition affected participants' accuracy, the hit was larger when Thematically related item inhibited Taxonomic processing, than vice versa. Critically, participants were also faster in Thematic than in Taxonomic task. This was true for both Neutral and Conflict condition. Since the effects on speed and accuracy of processing were found in a simple, strategyconstrained task, these results suggest that Thematic relations may be more salient than Taxonomic relations even in adulthood. If thematic relations are activated faster they may 
be hard to inhibit and thus they affect processing even in the task when participants are explicitly required to rely on taxonomic relations.

Experiment 3: Eye tracking study of conceptual preference

\section{Participants}

We recruited 32 students, all first-year undergraduates and native speakers of Serbian with normal hearing and normal or corrected-to-normal vision. All participants gave written informed consent prior to the study. Three of the recruited students were excluded from the analysis due to the failure of calibration.

\section{Stimuli}

We used the same stimuli set used in Experiment 1 and Experiment 2 (Table 1 in Appendix).

\section{Procedure}

We used the standard matching-to-sample task. Participants were presented with an auditory cue (e.g. "monkey"), followed by simultaneous presentation of three visual items, one thematically related (e.g. "banana"), one taxonomically related (e.g. "giraffe") and the third unrelated to the preceding word (e.g. "bench") (see Figure 1).

The instruction was neutral: Participants were instructed to choose the object that was best related to the auditory prime. The instruction, provided in Serbian, translates approximately to: "Your task is to decide which of the objects in each picture is most closely related to the named object." Stimulus position was counterbalanced throughout, and stimulus set presentation order was randomized.

\section{Experimental setup for eye-tracking experiments}


Participants were tested individually, seated approximately $100 \mathrm{~cm}$ from the computer display. Visual stimuli were presented on a CRT monitor screen (ViewSonic G90fB CRT, 19", $1600 \times 1200$ pixels, $36.5 \times 27.5 \mathrm{~cm}$, subtending a visual angle of $20^{\circ} \times$ $15^{\circ}$, running at $77 \mathrm{~Hz}$ ) using the SR Research Eyelink Experiment Builder software (SR Research Ltd., Mississauga, Ontario, Canada). Auditory stimuli were presented via two loudspeakers positioned behind the viewing monitor. Sound level was fixed at approximately $60 \mathrm{db}$.

Eye movements were registered using an EyeLink II head-mounted eye-tracker system (SR Research Ltd., Mississauga, Ontario, Canada) sampling at $500 \mathrm{~Hz}$ from the right eye. Viewing was binocular. For calibration, we used a standard nine-point fixation array. After half of the trials, the eye-tracker was recalibrated using the same fixation array. If the validation was poor, the calibration process was repeated. Calibration took typically about $90 \mathrm{~s}$. Trial initiation was manually controlled. Before each trial, in order to allow eyetracker drift correction, participants were asked to fixate a single, centrally located dot. After participants successfully fixated the dot, the experimenter pressed a button to initiate the next trial. The trial was terminated by participant response. Eye movements were recorded from the beginning of each trial until the participant pressed the joystick button. Responses were recorded using a SR Research gamepad. The entire experiment lasted 10 minutes on average.

\section{Areas of interest}

Rectangular areas of interest (AOI) of identical size $(200 \times 200$ pixels $)$ were drawn around each object in the display. Fixations were counted for each object type (thematic, taxonomic, and unrelated) when they landed within the boundaries of the corresponding AOI, which did not overlap.

\section{Measurements}

We recorded participants' choice and derived five measures from eye-tracking data: first fixation position, number of fixations, percentage of fixations, looking (fixation) time 
and percentage of looking (fixation) time. Data were analyzed in $3 \times 3$ repeated measures analyses of variance with Stimuli Type (Thematic, Taxonomic, Unrelated) and Stimuli Position (Left, Top, and Right) as within-subject factors.

\section{Results}

Participants' choice

Participants consistently chose the Thematic relationship over the Taxonomic relationship and the Unrelated option for all 24 out of the 24 triads used in the study $\left(\chi^{2}(2)\right.$ $=934.51, p<.01)$. Even for trials that received the greatest proportion of Taxonomic responses, the Taxonomic / Thematic ratio did not exceed 2/3, and Thematic choices made up two thirds or less of all choices in only of three of the 29 participants.

Before analysis, eye-tracking data were scanned for missing values. Only three trials had no eye-movement data due to participants fixating none of the regions of interest. Missing data points were treated as 0s. Since approximately $180 \mathrm{~ms}$ is needed for a saccade to be initiated (see Huetting \& Altmann, 2004), all fixation data recorded in the first $200 \mathrm{~ms}$ after visual stimulus presentation were dismissed.

\section{The first look}

The first look was significantly affected by Stimulus Type $\left(\chi^{2}(2)=16.16, p<.01\right)$, such that participants directed their first look more often towards Taxonomically than Thematically related objects $\left(\chi^{2}(1)=6.09, p=.01\right)$.

\section{Percentage of looking time and looking time (in ms)}

We found a main effect of Stimulus Type on the percentage of total looking time dedicated to each image and the amount of time (in ms) spent looking at a each image. Post-hoc tests for both variables showed that participants preferred Taxonomically related over Unrelated objects, but also Thematically related objects over Taxonomically related ones (Table 4). 


\section{Percentage of fixations and the number of fixations}

Analysis of the number of fixations and the percentage of fixations falling on the regions of interest revealed a significant main effect of Stimulus Type and a main effect of Stimulus Position. Both measures had significantly greater value for Taxonomically related than Unrelated objects, and for Thematically related than Taxonomically related ones. In terms of simple location effects independent of object relatedness status, fixations were equally distributed between Left and Top position, but the number and the percentage of fixations were significantly lower for the Right position in comparison to the two others.

Table 4. Differences in looking time and number of fixations by relatedness

\begin{tabular}{lll}
\hline & ANOVA & Comparisons (Post-hoc, Bonferroni) \\
\hline Looking time (percentage) & $F_{1.7,47.3}=116.90, \eta^{2}=.807$ & Thematic $>$ Taxonomic $>$ Unrelated \\
Looking time (in ms) & $F_{1.7,47.9}=97.20, \eta^{2}=.776$ & Thematic $>$ Taxonomic $>$ Unrelated \\
\hline Number of fixations & $F_{2,56}=77.71, \eta^{2}=.735$ & Thematic $>$ Taxonomic $>$ Unrelated \\
(percentage) & $F_{2,56}=6.96, \eta^{2}=.199$ & Left $=$ Top $>$ Right \\
& $F_{2,56}=77.58, \eta^{2}=.735$ & Thematic $>$ Taxonomic $>$ Unrelated \\
Number of fixations & $F_{2,56}=10.30, \eta^{2}=.269$ & left=top $>$ right \\
\hline
\end{tabular}

* all $\mathrm{F}$ tests and Post-hoc tests were significant at a threshold of $p<0.01$, with the exception of the contrast in the number of fixations between the left and right position significant at $p<.05$.

a.

b. 

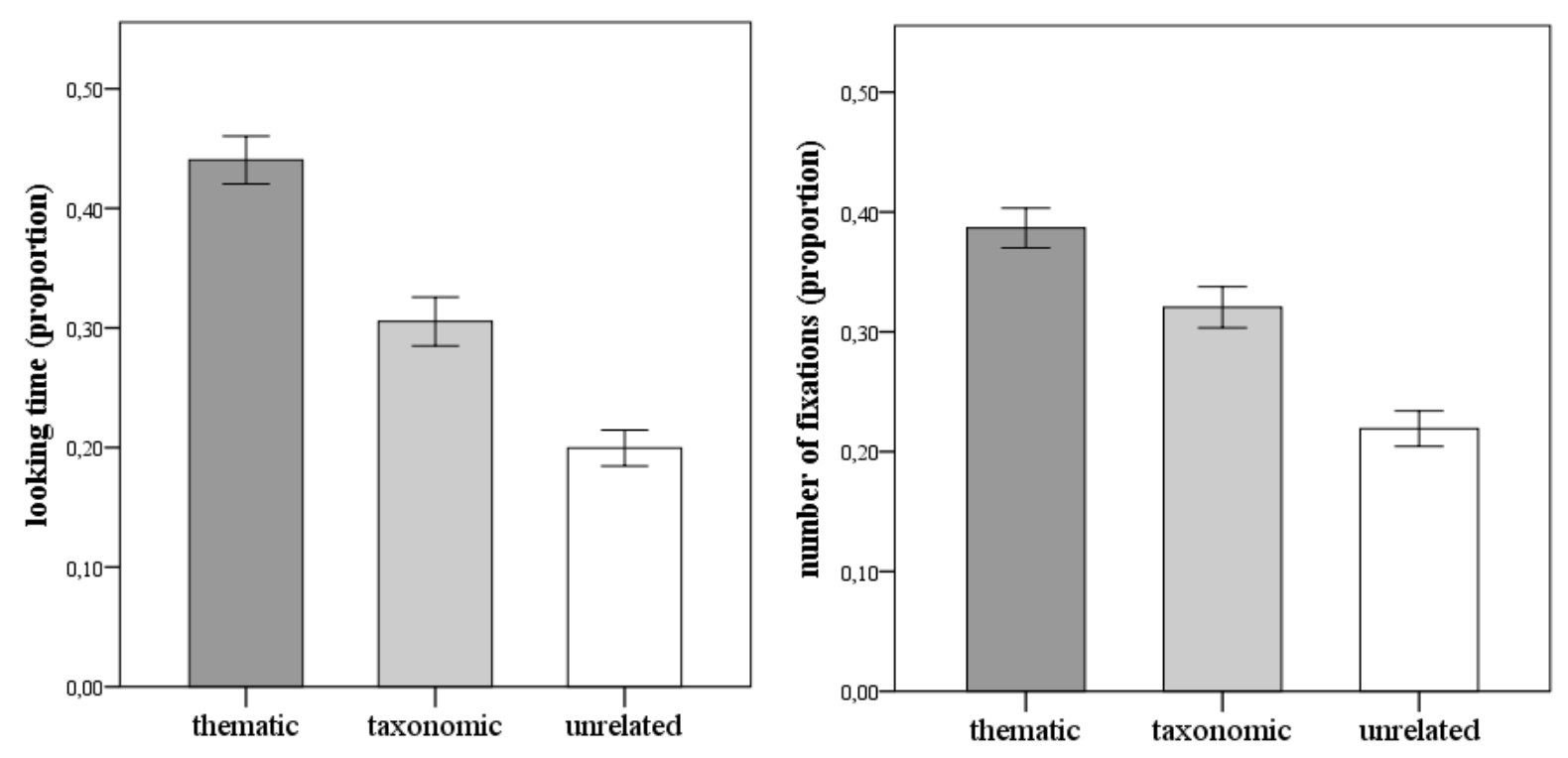

Figure 4. Average looking time (left) and average number of fixations (right). Error bars represent confidence intervals.

\section{General Discussion}

We report findings of three experiments designed to determine whether conceptual preference of adults in matching tasks is a matter of strategy or one of conceptual salience.

In Experiment 1 and Experiment 2, we used a guided matching task in which participants were not free to choose a particular relationship, but rather instructed to only attend one. The logic behind the design of these experiments was that salience of the competing conceptual relation should influence the ease with which the target relationship is processed. Thus, if thematic relationships are more salient, participants should find it more demanding (and thus be slower and less accurate) when matching objects taxonomically, since the thematic alternative would inhibit the taxonomic option, and reciprocally. We found exactly that: Differences in accuracy and speed between conditions suggested that reliance on taxonomic relationships during categorization is harder than categorization based on thematic relationships. These results are consistent with the results from other studies (Bassok \& Medin, 1997; Wisniewski \& Bassok, 1999; Gentner \& Brem, 
1999; Lin \& Murphy, 2001) showing that thematic and taxonomic relationships are intrusive and activated even when they are not relevant or are even counter-productive.

In Experiment 3, educated young adults shown strong thematic preference in a standard matching task under the neutral instruction of finding the "object that is most closely related to the target". We thus tested if there was more to conceptual preference than just strategy, by examining preference on measures of conceptual processing that are more subtle than participant selection under instruction.

Over and above a simple object position effect, presumably driven by direction of reading and checking strategy (left and top position having attracted more fixations than rightmost location), participants tended to fixate the taxonomic option first. For example, upon hearing the word monkey, they tended to first fixate a picture of a giraffe, then that of a banana, and then that of a bench. The early preference for taxonomically related items is unexpected under the assumption of conceptual preference for thematic relations. One possible explanation would be that taxonomic relations represent a more salient type of semantic information, and preference in selection of thematic options is strategic. However, as previously suggested by some authors (e.g. Lin \& Murphy, 2001), in studies using pictorial stimuli, taxonomic items might receive more attention due to visual similarity between the taxonomically related objects. Since it is impossible to tease apart the effects of visual similarity and semantic relatedness on the first look, we believe it is useful to view this finding in the context of other eye-movement measures. Critically, the first fixation was the only measure of eye-movement behavior for which taxonomic relations had an advantage over thematic relations. Taking into account the whole period from visual stimulation to decision-making and button pressing, thematically related objects received significantly more attention than taxonomically related items and the unrelated ones. We characterized three relationship types from all four eye-tracking measures: The thematic option received the most attention, the taxonomic option significantly less, and the unrelated object the least. 
It is important to note that thematic items received more attention despite the fact that taxonomic relations were likely boosted by the presentation of items as pictures (Huetting \& Altmann, 2004; Lin \& Murphy, 2001). Even though the first fixation appeared to be guided by taxonomic relation (or visual similarity), all other measures pointed to a preference for thematic relations. Thus, eye-tracking study supports findings of Experiment 1 and Experiment 2, by showing that thematic conceptual preference can be detected even before participants press a button and choose an option. Taken together, patterns of results presented here show that despite both types of semantic information, thematic and taxonomic, are activated and competing during a matching process, thematic information is overall more salient than taxonomic information.

Future studies may want to supplement the categorization performance with participants' justification of choice, i.e. participants could be explicitly asked about their strategies for the task. Participants' explanations may further enrich our understanding of the mechanisms responsible for the differences in performance.

\section{Conclusion}

In the three experiments presented here, we demonstrated the importance of thematic relations in adulthood. University students were faster and more successful when asked to match items based on thematic relations in comparison to matching items based on taxonomic relations (Experiment 1 and Experiment 2). Furthermore, they consistently looked longer and more frequently at thematically related objects than taxonomically related objects in the matching task (Experiment 3). Hence, our results suggest that thematic preference is not merely a response strategy. Rather, it reflects a spontaneous trend that affects early stages of stimulus evaluation. Our findings contribute to a growing body of literature that suggests that semantic relations based on object co-occurrence (i.e. thematic relations), which are dominant in early semantic development, continue to significantly influence cognitive processing in adulthood (Lin \& Murphy, 2001; Huettig et al, 2006; Yee, Overton \& Thompson-Schill, 2009). Moreover, our findings speak in favor 
of the assumption that even in adulthood, co-occurrence based relations remain more salient than relations based on object similarity (i.e. taxonomic relations).

\section{Conflict of Interest}

The authors declare that the research was conducted in the absence of any commercial or financial relationships that could be construed as a potential conflict of interest.

\section{Author Contributions}

OS, VK and GT designed the experimental paradigm. Data collection was conducted by OS, and analysis was performed by OS, GT and VK. The manuscript was written by OS, GT and VK.

\section{Acknowledgments}

We would like to thank Aleksandra Ilić for proofreading the manuscript.

\section{Funding}

We are grateful to the Ministry of Education, Science and Technological Development of the Republic of Serbia for financial support through grant number 179033. 


\section{References}

Adlington, R. L., Laws, K. R., \& Gale, T. M. (2008). The Hatfield Image Test (HIT): a new picture test and norms for experimental and clinical use. Journal of Clinical and Experimental Neuropsychology.

Bassok, M., \& Medin, D. L. (1997). Birds of a feather flock together: Similarity judgments with semantically rich stimuli. Journal of Memory and Language, 36, 311-336.

Boersma, P. \& Weenink, D. (2009). Praat: doing phonetics by computer (Version 5.1.05) [Computer program]. Retrieved from http://www.praat.org/

Brown, R., \& Berko, J. (1960). Word Association and the acquisition of grammar. Child Development, 31, 1-14.

Chen, Q., Lei, Y., Li, P., Xi, L., Li, F., \& Li, H. (2013). How do taxonomic versus thematic relations impact similarity and difference judgments? An ERP study. International Journal of Psychophysiology, 90(2), 135-142.

Chiu, L.-H. (1972). A cross-cultural comparison of cognitive styles in Chinese and American children. International Journal of Psychology,7(4), 235-242.

Denney, N., \& Ziobrowski, M. (1972) Developmental changes in clustering criteria. Journal of Experimental Child Psychology, 13, 275-282.

Ervin, S. M. (1961). Changes with age in the verbal determinants of word association. American Journal of Psychology, 74, 361-372.

Estes, Z., Golonka, S., \& Jones, L. L. (2011). Thematic thinking: The apprehension and consequences of thematic relations. Psychology of Learning and Motivation, 54:249-294.

Gentner, D. \& Brem, S. K. (1999). Is snow really similar to a shovel? Distinguishing similarity from thematic relatedness. Proceedings of the 21st Annual Conference of the Cognitive Science Society. (pp. 179-184). Hillsdale, NJ: Lawrence Earlbaum Associates. 
Huettig, F., Quinlan, P.T., McDonald, S.A., \& Altmann, G.T.M. (2006) Models of highdimensional semantic space predict language-mediated eye movements in the visual world. Acta Psychologica, 121, 65-80.

Huettig, F. \& Altmann G.T.M. (2005). Word meaning and the control of eye fixation: semantic competitor effects and the visual world paradigm. Cognition, 96, B23-B32 Huettig, F., \& Altmann, G. T. M. (2004). The online processing of ambiguous and unambiguous words in context: Evidence from head-mounted eye-tracking. In M.

Ilić, O. (2015) The nature of conceptual knowledge: The role of thematic and taxonomic knowledge in the organization of semantic memory. [dissertation]. [Belgrade (Serbia)]: University of Belgrade.

Inhelder, B., \& Piaget, J. (1964). The early growth of logic in the child. New York: Norton. Ji, L., Zhang, Z., \& Nisbett, R. E. (2004). Is it culture or is it language? Examination of language effects in cross-cultural research on categorization. Journal of Personality and Social Psychology, 87, 57-65.

Kalénine, S., \& Bonthoux, F. (2006). The formation of living and non-living superordinate concepts as a function of individual differences. Current Psychology Letters, 19(2).

Kalénine S., Mirman D., Middleton E.L., \& Buxbaum L.J. (2012). Temporal dynamics of activation of thematic and functional knowledge during conceptual processing of manipulable artifacts. Journal of Experimental Psychology: Learning, Memory, and Cognition, 38 (5), 1274-1295.

Lin, E. L. \& Murphy, G. L. (2001). Thematic relations in adults' concepts. Journal of Experimental Psychology: General, 130, 3-28.

Lucariello, J., Kyratzis, A., \& Nelson, K. (1992). Taxonomic knowledge: What kind and when? Child Development, 63, 978-998. 
Luria, A. R. (1976). Cognitive development: Its cultural and social foundations.

Cambridge, MA: Harvard University Press.

Markman, E. M., Cox, B., \& Machida, S. (1981). The standard object-sorting task as a measure of conceptual organization. Developmental Psychology, 17, 115-117.

Mirman, D., Landrigan, J.F., \& Britt, A.E. (2017). Taxonomic and Thematic Semantic Systems. Psychological Bulletin, 143(5), 499-520.

Murphy, G. L. (2001). Causes of taxonomic sorting by adults: A test of the thematic-totaxonomic shift. Psychonomic Bulletin \& Review, 8, 834-839.

Nelson, K. (1977). The syntagmatic-paradigmatic shift revisited: A review of research and theory. Psychological Bulletin, 84, 93-116.

Osborne, J. G., \& Calhoun, D. O. (1998). Themes, taxons, and trial types in children's matching to sample: Methodological considerations. Journal of Experimental Child Psychology, 68, 35-50.

Pluciennicka, E., Coello, Y., \& Kalénine, S., (2013). Development of thematic, specific function, and general function relation processing during childhood: An eye- tracking study. 7th Workshop Concepts, Actions and Objects: Functional and Neural Perspectives.

Savic, O., Savic, A. M., \& Kovic, V. (2017). Comparing the temporal dynamics of thematic and taxonomic processing using event-related potentials. PLOS ONE, 12(12).

Slowiaczek, L. M., \& Pisoni, D. B. (1986). Effects of phonological similarity on priming in auditory lexical decision. Memory \& Cognition, 14, 230-237.

Smiley, S. S. \& Brown, A. L. (1979). Conceptual preference for thematic or taxonomic relations: A nonmonotonic age trend from preschool to old age. Journal of Experimental Child Psychology, 28, 249-257.

Unsworth, S.J., Sears, C.R., \& Pexman, P.M. (2005). Cultural influences on categorization processes. Journal of Cross-Cultural Psychology, 36, 662-688. 
Vygotsky, L. S. (1962). Thought and language. Cambridge, MA: MIT Press.

Waxman, S. R. \& Gelman, R. (1986). Preschoolers' use of superordinate relations in classiflcation and language. Cognitive Development, 1, 139-156.

Wisniewski, E. J. \& Bassok, M. (1999). What makes a man similar to a tie? Stimulus compatibility with comparison and integration. Cognitive Psychology, 39, 208-238.

Yee, E., Overton, E., \& Thompson-Schill, S. (2009). Looking for Meaning: Eye Movements Are Sensitive to Overlaping Semantic Features, Not Association. Psychonomic Bulletin and Review, 16(5), 869-874. 
Table 1. The full list of stimuli used in Experiment $1(a, b$ and $c)$ and Experiment 2

\begin{tabular}{|c|c|c|c|c|}
\hline \multirow{2}{*}{\multicolumn{2}{|c|}{ auditory prime }} & \multicolumn{3}{|c|}{ visual stimuli } \\
\hline & & thematic & taxonomic & unrelated \\
\hline \multicolumn{5}{|c|}{ practice trials } \\
\hline 1 & flower & vase & tree & penguin \\
\hline 2 & coconut & palm tree & pineapple & frog \\
\hline \multicolumn{5}{|c|}{ test trials } \\
\hline 1 & horse & saddle & zebra & hat \\
\hline 2 & hen & egg & sparrow & balloon \\
\hline 3 & cow & milk & donkey & notebook \\
\hline 4 & worm & apple & snail & chair \\
\hline 5 & chalk & blackboard & pencil & pear \\
\hline 6 & belt & pants & suspenders & cat \\
\hline 7 & swallow & nest & goose & trumpet \\
\hline 8 & boat & oars & speedboat & teacup \\
\hline 9 & hammer & nail & pliers & Christmas tree \\
\hline 10 & bee & honey & butterfly & bicycle \\
\hline 11 & pearl & shell & diamond & bread \\
\hline 12 & aquarium & fish & cage & glasses \\
\hline 13 & monkey & banana & giraffe & bench \\
\hline 14 & scissors & paper & saw & umbrella \\
\hline 15 & mouse & cheese & fox & pumpkin \\
\hline 16 & $d o g$ & bone & leopard & pipe \\
\hline 17 & shoelace & shoe & rope & grapes \\
\hline 18 & pistol & bullet & cannon & spoon \\
\hline 19 & elephant & peanuts & camel & paprika \\
\hline 20 & squirrel & hazelnut & kangaroo & pan \\
\hline 21 & rabbit & carrot & lion & guitar \\
\hline 22 & drums & drumsticks & saxophone & wardrobe \\
\hline 23 & shirt & tie & jacket & tambourine \\
\hline 24 & toothbrush & toothpaste & brush & bed \\
\hline
\end{tabular}


Instructions used in the relatedness judgment task

The instructions were based on the definitions of thematic and taxonomic categories found in Estes et al. (2011).

Thematic relatedness

Thematic relation is temporal, spatial, causal, or functional relation between things. More specifically, objects are thematically related if they perform complementary roles in the same scenario or event.

Examples of thematically related objects: plane - pilot, flower - vase.

Taxonomic relatedness

Taxonomic relatedness is relatedness between objects that share features and hence belong to the same semantic category.

For example, beaver and chinchilla are animals, apple and pear are fruit. 
The distributions of accuracy rates across stimuli sets in the three tasks

As it can be seen in Figure 1, errors are spread evenly across stimulus sets in all three tasks, and there are no extreme outliers. The two stimulus sets that had lowest accuracy in the taxonomic condition were: a) pearl, shell (thematic), diamond (taxonomic), bread (unrelated); and b) hammer, nail (thematic), pliers (taxonomic), Christmas tree (unrelated). These two stimuli sets do not share the problem of the potential confusion of the taxonomic match and the distractor. This is also evident in the type of the errors made for these two sets, which were predominantly thematic (.81 for pearl; 1.00 for hammer). In the case of the aquarium set, the same was true. When participants were making errors in the taxonomic condition, they were choosing the thematic pairs in $92 \%$ of the cases. This suggests that the accuracy differences dominantly result from the competition between the thematic and taxonomic competitors, rather than the competition between related items and distractors.

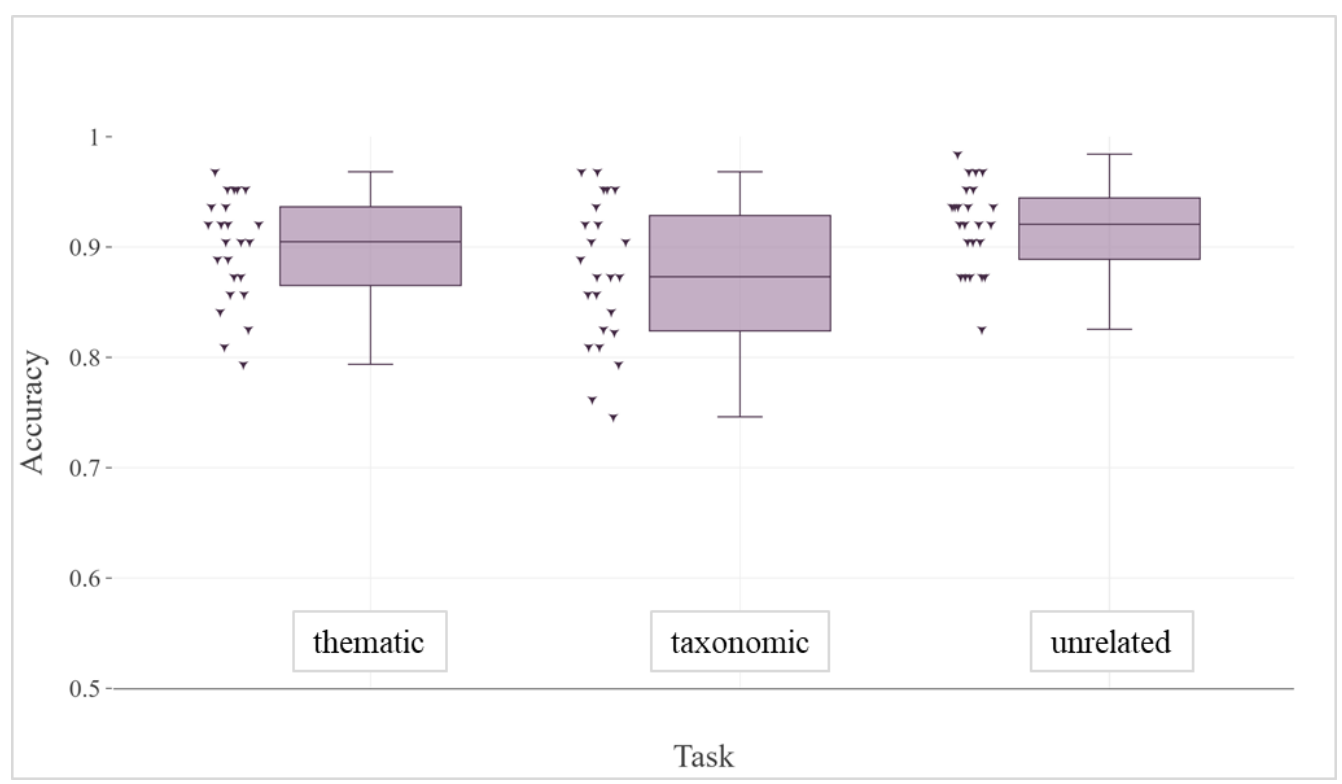

Figure 1. Box-plot diagram with Beeswarm plots showing the distributions of accuracy rates across stimuli sets in the three tasks. Data were collapsed across the three Experiments since there was no significant effect of the Experiment neither as a main effect nor in the interaction with the Task. 